\title{
LA EDUCACION PARVULARIA EN LA UNIVERSIDAD DE CHILE: UN COMPROMISO PAÍS
}

THE EARLY CHILDHOOD EDUCATION AT THE UNIVERSITY OF CHILE:

\author{
A COUNTRY COMMITMENT
}

Reapertura Educación Parvularia

\author{
Mónica Manhey Moreno \\ Especialista en: Educación Infantil \\ Depto. Educación. Universidad de Chile \\ Ignacio Carrera Pinto 1045. \\ mmanhey@u.uchile.cl
}

\section{Resumen}

El presente escrito relata la generación de la nueva carrera de Educación Parvularia en la Universidad de Chile, sus antecedentes históricos, elementos que se tomaron en cuenta para su diseño, sus énfasis y características que se aprecian como innovadoras, y finalmente la propuesta plasmada en el plan de estudios de manera sintética y algunos desafíos, los que se vislumbran no sólo para la carrera en la Universidad de Chile, sino como país. Es importante mencionar que se ha recogido información del trabajo sistemático realizado, por más de un año y medio, por un equipo de docentes, educadoras de párvulos, profesionales de la Universidad de Chile, que estuvo asesorado por expertos de la Red de Innovación de esta Universidad. Este informe fue el referente para la propuesta de apertura de la carrera.

Palabras clave: carrera - educación parvularia.

\begin{abstract}
The article describes the creation process of the new career in early childhood education at the University of Chile, including its historical background, the elements taken on carrying out its design, the innovative emphasis and features, and finally a synthesis of the proposal reflected in the curriculum. Challenges are discussed, not only for the University but also for the country. The information for this article has been gathered from the work carried out, more than a year and a half, by a team of teachers, early childhood educators,
\end{abstract}




\section{LA EDUCACION PARVULARIA EN LA UNIVERSIDAD DE CHILE: UN COMPROMISO PAÍS}

professionals of the Universidad de Chile, who were counselled by the experts of the Innovation Net. This was the reference for the proposal to initiate the career.

keyword: career - early childhood education

\section{INTRODUCCIÓN}

El año 2015, la Facultad de Ciencias Sociales de la Universidad de Chile, ha abierto nuevamente la carrera de Educación Parvularia, siendo un hito no sólo para la Facultad, sino para Universidad de Chile y nuestro país.

Este artículo toma como gran referente el trabajo que se desarrolló durante un año y medio; precisamente desde enero 2013 a agosto 2015 (Universidad de Chile 2014) por una comisión destinada a estudiar y diseñar la propuesta académica. Esta comisión estuvo constituida por académicos, educadoras, estudiantes y miembros del equipo de la red de innovación curricular de la Universidad de Chile. (1)

Antes de abordar lo que se refiere específicamente a la reapertura de la carrera de Pedagogía en Educación Parvularia, se hará una breve reseña histórica, posteriormente se presentan algunos elementos relevantes que aportaron a la construcción de la misma y, posteriormente, algunas características de la propuesta implementada y algunos desafíos.

Esta propuesta busca establecer un modelo de formación innovador que permita a la Universidad de Chile recobrar su liderazgo en la formación de Educadores/as de Párvulos en Chile, respondiendo al espíritu y misión de nuestra Casa de Estudios, a las tendencias para la formación de párvulos en el mundo y a los desafíos del país en este campo, por tanto, lo que se presenta no es sólo una "nueva carrera", sino una propuesta que contribuya al avance de la Educación Parvularia en el país. 


\section{LA EDUCACION PARVULARIA EN LA UNIVERSIDAD DE CHILE: UN COMPROMISO PAÍS}

\section{RESEÑA HISTÓRICA}

La Universidad de Chile ha estado vinculada al desarrollo de la educación nacional desde su fundación en el año 1842, lo que marca uno de los hitos más significativos de la naciente república, en aquellos años. "Representa en sí uno de los más claros actos de autodeterminación, al situar en el saber y la enseñanza pública el eje articulador del país que comienza a construirse.... “. (Serrano, 1993) De esta manera, el Estado le encargó ejercer labores de superintendencia en ese campo, y en 1889 le entregó la tuición del Instituto Pedagógico y de su cuerpo académico originario, conformado por ocho profesores alemanes. A lo largo del siglo XX, el Instituto Pedagógico de la Universidad de Chile fue el principal centro formador de docentes en el país y su evolución y desarrollo marcaron la educación chilena y latinoamericana. La Educación Parvularia en Universidad de Chile tiene una trascendente historia, en 1944 se crea la Escuela de Educadoras de Párvulos, bajo la rectoría de Don Juvenal Hernández, con el apoyo de dos destacadas mujeres feministas de la época, Amanda Labarca y la española Matilde Huici, todo ello llevó a relevar este nivel educativo y su saber pedagógico especializado (Peralta, 2013). Desde aquellos años la Universidad de Chile ha desempeñado un papel destacado en el ámbito nacional e internacional en la Educación Parvularia. Carrera que ha tenido ciertos hitos a lo largo del tiempo, unos que nos llenan de orgullo y otros que hicieron una gran sombra al despliegue de esta profesión dentro de la casa de Bello.

Con la Ley de Universidades de 1981, la carrera fue impactada por la nueva institucionalidad del Sistema de Educación Superior, establecido en época de dictadura en nuestro país, donde se cierra la carrera y pasa a la "Academia Superior de Ciencias Pedagógicas", la que en 1986, se convierte en la Universidad Metropolitana de Ciencias de la Educación, (UMCE).

En el año 1990 con la ratificación en nuestro país de la Convención Internacional de los Derechos del Niño, obliga al Estado y a las distintas instituciones públicas a asumir los compromisos de promoción y protección de los derechos de los niños y niñas y junto con el proceso de recuperación de la democracia, se abren puertas para volver a instalar la 


\section{LA EDUCACION PARVULARIA EN LA UNIVERSIDAD DE CHILE: UN COMPROMISO PAÍS}

Educación Parvularia en nuestra universidad. Es así como en el año 1998, consciente de la importancia de la educación infantil para el mejoramiento de la calidad de la educación, el Departamento de Educación de la Facultad de Ciencias Sociales, desarrolla un proyecto innovador liderado por la actual profesora emérita Dina Alarcón Quezada en conjunto con Alondra Díaz. Este proyecto estaba dirigido a aportar al problema de la transición y articulación entre Educación Parvularia y Educación Básica. Como respuesta a ello, se desarrolla una propuesta académica que abarca una formación para trabajar con niños entre 0 y 8 años, propuesta que implicó una innovación pedagógica para el país, siendo la primera carrera con dicho enfoque. Además, se proponen cursos diferentes a lo que hasta el momento se habían ofrecido en la formación como son Imaginario Infantil, Construcción de Ambientes de Aprendizaje y Práctica Reflexiva, entre otras muchas actividades curriculares.

Para este proyecto, se contó con el apoyo de la Organización de Estados Americanos (OEA) a través de su Agencia Interamericana para la Cooperación y el Desarrollo (Proyecto de Formación de Educadores de Infancia y la Innovación Pedagógica), la cual aportó los recursos necesarios para el contacto y traslado de especialistas en los temas innovadores, llevándose a efecto reuniones de estudio y talleres con especialistas de México, Chile y Colombia para académicos del programa, alumnas y directoras de los centros de práctica.

El año 2001, se da por finalizado el proyecto para hacer realidad la apertura de la carrera de Educación Parvularia y Básica Inicial, la que permanece hasta ahora. Es importante hacer mención que dicha carrera fue acreditada, por cinco años, a partir del año 2012. Sin embargo, después de una evaluación referida al campo laboral y a las necesidades específicas, así como a algunos problemas estructurales en el Departamento de Educación, se decide ofrecer las dos carreras como lo que hoy es noticia: una carrera de Pedagogía en Educación Básica, en la Facultad de Filosofía y otra, de Educación Parvularia, alojada en el Departamento de Educación de la Facultad de Ciencias Sociales. 


\section{LA REAPERTURA DE EDUCACIÓN PARVULARIA EN LA UNIVERSIDAD DE CHILE:}

En la Universidad de Chile, se firmó un Convenio de desempeño formulando un Plan de Mejoramiento Institucional (PMI), denominado “Formando Profesores para Chile”, de manera de devolver las pedagogías a esta importante Casa de Estudios, “instalando a la educación como una de sus áreas disciplinarias más relevantes, con el propósito de contribuir — desde el oficio académico — a la urgente demanda por mayor calidad y equidad para el sistema educacional chileno, y a la construcción del país futuro” (Universidad de Chile, 2012 p. 12).

Dicho Plan de Mejoramiento Institucional tuvo los siguientes objetivos:

1) formar docentes para ejercer de manera innovadora y generosa una de las profesiones más complejas e importantes de la sociedad.

2) ofrecer al Estado, la sociedad y otras universidades, un referente colaborativo para el mejoramiento educacional, a través de la reflexión y la investigación multidisciplinaria en el campo de la educación, con especial énfasis en el ámbito de lo público.

Como se hizo mención anteriormente, el diseño de la carrera no fue algo arbitrario ni simple, se debió desarrollar un trabajo de investigación, en función de qué elementos esenciales debía tener un plan formador de Educación Parvularia. El proceso se inicia en enero del año 2013, donde se estableció un cronograma de trabajo de acuerdo a innovación curricular. Para ello, se realizaron diferentes acciones. En primer lugar, se recogieron Necesidades Formativas y Compromisos Formativos. En cuanto a las necesidades formativas, se realizó una exhaustiva revisión de antecedentes, los que finalmente permitieron seleccionar los “Compromisos Formativos”, es decir, de todas las necesidades se escogería cuáles serían factibles de abordar desde la formación en nuestra Casa de Estudios a través de un Plan de Formación. A continuación, se describen brevemente las tareas llevadas a cabo: 
- Revisión de la literatura internacional especializada sobre formación de Educadores/as de párvulos. Al respecto se levantó información, por ejemplo, la heterogeneidad en los programas internacionales en la definición del grado académico asociado a la carrera de Educación Parvularia. En algunos, el título habilitante es de postgrado en modalidad de Magister en Educación. Los programas internacionales cuentan con plantas docentes, en su mayoría, integradas por especialistas con postgrados. Al mismo tiempo, se integran especialistas en el aula como tutores de práctica. En ocasiones, estos tutores sólo poseen el grado de educador y en otras la Maestría en Educación. En cambio, los programas nacionales se diferencian sustancialmente con respecto a los programas internacionales analizados, principalmente, en: (a) los requisitos para el ingreso a la carrera; (b) a la composición del cuerpo docente y; (c) la formación continua (otorgada por los programas de magíster en educación) (Universidad de Chile, 2014)

- Revisión del Estado del arte de la Educación Parvularia. Se revisó y se presentó en el informe que sustentó la creación de la carrera, desde antecedentes históricos de la Educación Parvularia en Chile, así como la definición que se ha establecido de este nivel educativo, sus niveles, tipos de programas y modalidades curriculares como de atención, la institucionalidad que la alberga y datos de cobertura hasta la fecha. Un aspecto relevante fueron los datos recogidos, en relación a la oferta vinculada con la formación de educadoras de párvulos, lo que demostró la gran heterogeneidad en propuestas curriculares. De ahí, emergen diversos aprendizajes posterior a la literatura revisada como a antecedentes empíricos, lo que demuestra que “...crece el consenso de que la formación de Educadores/as de Párvulos debe abarcar un rango de edad delimitado (tradicionalmente desde el nacimiento hasta los 6 años), con el fin de asegurar que los programas de formación puedan abordar con profundidad las distintas áreas que integran la carrera (Universidad de Chile, 2014). Esto último avaló la necesidad de una carrera específica para este nivel que además, tenga una duración de más de cuatro años, lo que según estudios de Fukkink y Lont (2007) (citado en 


\section{LA EDUCACION PARVULARIA EN LA UNIVERSIDAD DE CHILE: UN COMPROMISO PAÍS}

Universidad de Chile, 2014, p. 20) señalan que "existe un efecto estadísticamente significativo entre la formación superior especializada y las competencias de los/as Educadores/as de Párvulos”, siendo capaces de (a) desplegar prácticas pedagógicas más apropiadas a las características del desarrollo de los niños/as y niñas a su cargo, como señala cargo, (b) demostrar mayor sensibilidad a las necesidades de éstos, e (c) influir en la calidad del centro educativo en su conjunto.

Se realizaron entrevistas y grupos focales para conocer la opinión de diferentes agentes claves. En cuanto a las entrevistas, es de relevancia destacar que se recogieron las opiniones y expectativas de los empleadores de diversos programas de Educación Parvularia, tales como: Coordinadora de Proyectos Educativos de municipalidades, encargadas de educación parvularia en colegios particulares y subvencionados, Directora de Departamento Técnico de la Junta Nacional de Jardines Infantiles, así como de la Fundación Integra, y de otras instituciones que tienen gran número de educadoras a su cargo, así como encargadas nacionales de proyectos alternativos. Es importante hacer mención que la totalidad de los empleadores expresó altas expectativas respecto al trabajo a realizar por las Educadores de Párvulos; esperando que éstas tengan competencias sólidas, dada la importancia de la labor que deben llevar cabo. También se les hizo entrevistas semiestructuradas a egresadas de la carrera de Educación Parvularia y Básica Inicial de la Universidad de Chile donde destacaron la relevancia de tener una infraestructura, equipamiento y recursos adecuados para la formación tales como material de biblioteca especializado en educación infantil, también comentaron con respecto a la importancia de las prácticas, su acompañamiento y retroalimentación y la importancia de docentes que sean educadores y hayan tenido experiencia en aula y la necesidad de potenciar el trabajo con niños y niñas menores de 3 años. Estas y otras observaciones fueron importantes insumos para la elaboración posterior del proyecto de la carrera a implementar.

Con respecto a los grupos focales, se llevaron a cabo ocho grupos focales con Educadoras, Técnicos en Educación Parvularia, apoderados nivel socio económico medio, apoderados nivel socio económico alto, apoderados nivel socio económico 
bajo y académicos que conformaron la comisión de creación de la carrera. Los aportes fueron relevantes, especialmente referidos al tipo de educador que aspiran los apoderados, destacando el ámbito actitudinal: sensibles, cariñosos, empáticos y las educadoras se refirieron a su rol y la importancia del trabajo en equipo entre otros aportes.

- Análisis de la oferta formativa de las carreras de Educación Parvularia o Educación Inicial en otros países. Los países estudiados fueron los siguientes: en Cuba, el Programa de Educación Preescolar de la Universidad de Ciencias Pedagógicas Enrique José Varona. Esta decisión se avaló en su reconocimiento a nivel mundial por UNESCO (Casassus, Cusato, Froemel, \& Palafox, 2000 en Universidad de Chile, 2014, p. 35). Asimismo, se estudió, en Estados Unidos, el programa de Wheelock College, localizado en Boston; su elección se debió por ser reconocido en la literatura especializada como un programa ejemplar de formación, según la opinión de los empleadores de ese país (Darling-Hammond, 2006), un país nórdico como Finlandia y la Universidad de Oulu, su selección fue dada la difusión de los buenos resultados académicos obtenidos por este país. Otro país que se estudió fue Australia y la Universidad de Monash, considerando que esta universidad ha ocupado el lugar número 6 de las mejores universidades del mundo, de acuerdo al ranking 2013 "QS World University Rankings" en cuanto a su reputación académica (QS Quacquarelli Symonds Limited, 2013). Finalmente, mencionamos a Inglaterra que fue estudiada por su programa prestigioso desarrollado desde el Instituto de Educación de la Universidad de Londres, donde existen connotados académicos reconocidos a nivel internacional como Peter Moss y Gunila Dahlberg que han realizado importantes aportes.

\section{- Análisis de la oferta formativa de las carreras de Educación Parvularia en Chile.} Se estudiaron universidades que contemplaran los siguientes criterios: (a) representar a universidades del CRUCH y privadas; (b) estar localizados en Santiago y regiones; y 
(c) contar con acreditación de la carrera. También se analizó cómo se ha expandido la oferta en la formación de educadoras a través de diferentes casas de estudio (más de 43) y sedes en regiones. Además, se pudo desprender de la investigación que de las universidades estudiadas, existen distintos programas de Educación Parvularia.

o Educación Parvularia: con una formación acotada a dicho nivel educativo y sin menciones de especialidad;

o Educación Parvularia con mención/idioma: con una formación acotada a dicho nivel educativo pero que incorpora diversas menciones como la artística o la opción de cursarla con especialización bilingüe; y

o Educación Parvularia y Básica Inicial: que abarca desde el nivel de Educación Parvularia hasta los primeros años de Educación Básica y originalmente en Universidad de Chile, aun cuando en forma posterior existieron un par de ofertas con dichas características.

- Revisión de antecedentes internos dentro de la Universidad. Se tomó como referencia tanto el informe de acreditación de la carrera de Educación Parvularia y Básica Inicial del Departamento de Educación, que otorgaba una acreditación de cinco años, así como sus antecedentes y el contexto institucional al interior de la Universidad de Chile. Al respecto fue un gran aporte los testimonios ofrecidos por las egresadas y estudiantes de la carrera de Educación Parvularia y Básica Inicial ofrecidos mediante las entrevistas como se comentó en párrafos anteriores.

- Campo Laboral de los/as Educadores/as de Párvulos. Al respecto, se pudo constatar que el campo laboral en su mayor parte se concentra en los establecimientos educacionales de modalidad convencional que proveen Educación Parvularia; sean éstos de dependencia municipal, particular subvencionada, particular pagada, además de las instituciones estatales dependientes de MINEDUC, JUNJI y de Fundación INTEGRA. Es de relevancia que justo durante el período de diseño de la carrera se encontraban en desarrollo algunos proyectos de ley en el país que permitirían potenciar el nivel de Educación Parvularia como es el caso de la carrera docente. 
- Revisión de la política pública y demandas sociales del país relativas a la Educación Parvularia. En los últimos años se han impulsado medidas de política pública orientadas a regular la formación inicial docente, las que también afectan la formación de Educadores/as de Párvulos. Entre estas medidas se encuentran: Prueba Inicia; Beca Vocación de Profesor; Proyecto de Ley Carrera Docente; Estándares Orientadores para Carreras de Educación Parvularia. Entendiendo que este último documento tiene como propósito guiar los programas de formación en torno a los conocimientos, habilidades y disposiciones profesionales con que deben contar las egresadas de las carreras de Educación Parvularia, permitió tener un punto de referencia para el desarrollo de la propuesta académica

- Jornadas de validación. Finalizada las etapa anteriores se hizo una primera jornada de socialización y validación del proceso, (junio 2013) presentado a un grupo de expertos convocados en un primer taller de validación donde se expuso el trabajo realizado a distintos actores de la comunidad especialistas en el campo de la Educación Parvularia: educadoras de párvulos en ejercicio, estudiantes de Educación Parvularia y Básica Inicial de la Universidad de Chile, académicos e investigadores de otras universidades, entre otros. En dicha ocasión se presentaron las necesidades formativas y los compromisos formativos, siendo estos últimos aquellos con los cuales la carrera se comprometería, lo que se constituyó en la base para la elaboración del perfil de egreso de la carrera. Paralelamente a ello, se recogieron los aportes de visitas de académicas internacionales (Iram Siraj, Universidad de Londres y Pamela Oberhuemer, del Instituto Estatal de Investigación en Primera Infancia, Alemania). Para la segunda jornada de validación (noviembre 2013), se convocó a las mismas personas de la jornada anterior, validando así el perfil de egreso así como los ámbitos, competencias y subcompetencias.

- Declaración sintética del perfil de egreso: Posterior a los resultados recogidos de las diferentes instancias de validación, se redactó el perfil de egreso lo que implica 


\section{LA EDUCACION PARVULARIA EN LA UNIVERSIDAD DE CHILE: UN COMPROMISO PAÍS}

una declaración identitaria que representa la racionalidad de la profesión enmarcado en el sello institucional:

La educadora de párvulos egresada de la Universidad de Chile es una profesional de la pedagogía, capaz de generar propuestas pedagógicas pertinentes que promuevan el desarrollo, el aprendizaje, el bienestar y la ciudadanía de los niños desde su nacimiento hasta los seis años de edad, considerando el juego como medio vital en esta etapa de la vida. Con un fuerte compromiso social, reconoce el rol de la comunidad educativa potenciando la participación de familias y de otros agentes y organizaciones relevantes en los proyectos educacionales en que participa. A través de la reflexión crítica sobre su campo profesional, contribuye a la construcción de nuevos saberes en Educación Parvularia, y en consecuencia, al desarrollo de su profesión.

Comprende a la educación como un fenómeno social y cultural en constante cambio, $y$, por ende, está comprometida a continuar su aprendizaje profesional a lo largo de su vida laboral, incorporando de forma pertinente las tecnologías de información y comunicación. Respeta y valora la diversidad y las diferencias individuales, reconociendo a los niños desde el nacimiento como sujetos de derecho y agentes de su propio aprendizaje. De la misma manera, se concibe a sí misma como co-constructora de procesos de aprendizaje, centrando su propuesta pedagógica en las características individuales de los niños, considerando el contexto histórico, socio-cultural, económico y político con el que ellos interactúan, y la variedad de recursos disponibles para su práctica pedagógica.

Cuenta con competencias claves para desempeñarse en los siguientes ámbitos, tanto en el sector público y privado, así como en distintos contextos socioculturales, modalidades curriculares y programas de atención.

- Definición de Ámbitos de desempeño: orientados por la definición de familias de problemáticas que dan cuenta de los compromisos formativos declarados en la fase de levantamiento de información. Respondiendo a ello los ámbitos definidos para la carrera producto de la literatura revisada, antecedentes empíricos y la participación de diferentes actores nacionales e internacionales quedaron los siguientes:

$\checkmark$ Niño, infancia y aprendizaje.

$\checkmark$ Familia y comunidad educativa.

$\checkmark$ Pedagogía, currículo y didáctica. 
$\checkmark$ Pensamiento Reflexivo y Métodos Investigación en el ámbito de la Pedagogía.

$\checkmark$ Atributos personales asociados al desarrollo profesional desde el campo científico con énfasis en las Ciencias Sociales.

$\checkmark$ Campo profesional.

- Competencias por ámbito: las que se visualizan como las actuaciones profesionales o disciplinares concretas que los egresados deberán demostrar al final del itinerario formativo.

- Definición de la estructura curricular y malla. La elaboración del plan de estudios de la carrera contempló el análisis de los desafíos que el perfil de egreso le plantea al plan de formación. Esto, sobre la base de criterios que orientan y caracterizan la organización de la propuesta curricular. Estos criterios se desprenden del modelo educativo de la Universidad de Chile y del reglamento de estudios de pregrado: flexibilidad, integración, movilidad, implicancias pedagógicas, articulación pre y post grado entre otros. Además, la eelaboración de mapa de tributación de competencias y subcompetencias, construyendo el trayecto de formación, que supone la elaboración de la matriz temporal, la instalación de los ámbitos de desempeño, de las competencias y sub-competencias declaradas, para finalmente definir las actividades curriculares, entendidas como las experiencias de aprendizaje definidas a partir de la agrupación de sub-competencias, todo ello de acuerdo al Sistema de Créditos Transferibles, (SCT) lo que dio finalmente 300 SCT en la carrera. Es importante hacer mención que se optó por ofrecer una amplia oferta de actividades curriculares como cursos; tanto de carácter obligatorio como electivos y talleres, en función de ciclos formativos o familias de problemas. Todo ello articulado con la práctica la que se da desde el primer semestre y en forma progresiva, favoreciendo entre las actividades académicas una articulación vertical como horizontal. Además las/educadoras en formación deberán tomar Cursos de Formación General que ofrece la Universidad y que todo estudiante de esta Casa de Estudios debe tomar, y que se orientan a desarrollar en el estudiante ciertas 
competencias genéricas importantes tanto en el desempeño profesional como en la actuación ciudadana.

Es relevante hacer mención que durante todo el proceso y, evidentemente, en su fase final, este trabajo fue presentado a las diferentes instancias de la Universidad de Chile, para que finalmente se presentara y se aprobara por unanimidad en el Senado Universitario el 28 de julio del año 2014.



Imagen 1 - El 28 julio 2014 se aprueba la carrera de Pedagogía en Educación Parvularia en la Universidad de Chile

\section{CARACTERISTICAS DISTINTIVAS DE LA CARRERA DE PEDAGOGÍA EN EDUCACION PARVULARIA EN LA UNIVERSIDAD DE CHILE}

Algunas de las características que son distintivas de la carrera de Pedagogía en Educación Parvularia:

Por encontrase en la Universidad de Chile:

- Admisión: Se exigen como mínimo 600 puntos ponderados para postular vía PSU, (Prueba de Selección Universitaria) lo cual es un requisito para ingresar a cualquier carrera de la Universidad de Chile. Sin embargo, la Universidad dispone de vacantes denominadas SIPEE, lo que es una modalidad de acceso para estudiantes de excelencia, egresados de establecimientos públicos o de dependencia municipal, 


\section{LA EDUCACION PARVULARIA EN LA UNIVERSIDAD DE CHILE: UN COMPROMISO PAÍS}

que no alcanzan el puntaje necesario para ingresar de manera tradicional y que pertenecen a los tres quintiles de ingresos, así como tener un promedio de 5,5 entre primero y tercero de Enseñanza Media, entre otros requisitos. (www.becasycreditos.cl). También existen los ingresos especiales, tanto vía transferencia interna o por transferencia externa. Es importante hacer mención que de la oferta que se da en Chile, en relación a los requisitos para postular a los programas que ofrecen las universidades, "se tiene que el 58,1\% de los programas pide Puntaje de Corte en el proceso de admisión, y un 20,9\% requiere sólo la PSU rendida. En tanto, el 21\% restante se distribuye en otros tipos de requisitos” (puntaje mínimo de 450 o 500 puntos en PSU, y sin requisitos 4,65\% (univ. De Chile, 2014, p.44)

- Sistema de Créditos Transferibles: Este es "un sistema en el cual al volumen total de estudio efectuado por un estudiante (tiempo de docencia directa más tiempo de estudio independiente) se le asigna un valor numérico...”. (Bisschop, 2006, p. 4) Coherente con ello, la propuesta formativa de la carrera de Pedagogía en Educación Parvularia adhiere plenamente al Sistema de Créditos Transferibles (SCT) con la finalidad de favorecer la transversalidad de la formación tanto entre las unidades académicas de la Universidad como a nivel de pre y postgrado. Este sistema está centrado en el estudiante, basándose en la carga de trabajo, de manera de vincularse directamente con las competencias y resultados de aprendizaje. Esta carrera se ajusta a 300 créditos anuales divididos en 10 semestres y en cada uno con un total de 30 créditos.

- Modelo por competencias. El modelo educativo de la Universidad de Chile se caracteriza por adoptar un enfoque curricular basado en competencias y un perfil profesional acorde con necesidades-país, entendiendo las competencias como:

...conjuntos dinámicos e integrados de conocimientos, habilidades y actitudes, que pueden ser inducidos durante el proceso formativo y cuyo grado de adquisición y/o desarrollo (logro de aprendizaje) es susceptible de evaluarse; las competencias, que están al servicio del perfil de egreso, 
requieren metodologías docentes renovadas cuyo centro es el estudiante. (Universidad de Chile, 2010, p. 9).

- Certificación Intermedia y/o Complementaria: deberá ser solicitada y tramitada por el estudiante en la Secretaría de Estudios correspondiente a su Facultad.

- Formación en Inglés: Todos los estudiantes de la Universidad deben rendir el Test de Diagnóstico de Inglés, independiente del nivel de inglés que posean al momento de matricularse. La Facultad de Ciencias Sociales no es la excepció; así, al obtener el cuarto nivel de inglés, todos pueden optar a una certificación de reconocimiento internacional.

- Movilidad estudiantil: Permite a estudiantes extranjeros de pregrado la oportunidad de realizar estudios en esta Institución. Este programa está destinado estudiantes que provienen de universidades extranjeras con las cuales la Universidad ha establecido un convenio de mutuo apoyo a la docencia. Estos estudiantes pueden cursar uno o dos semestres en la Universidad y, aun cuando son evaluados, mantienen su vinculación con sus universidades de origen. Del mismo modo, está el Subprograma de Estudios Parciales en el Extranjero, a través del cual estudiantes de la Universidad de Chile cursan algunas asignaturas en una universidad extranjera con la que se tenga convenio y para lo cual reciben una beca parcial de estudios

\section{Específicos de la carrera:}

- Estar en la Facultad de Ciencias Sociales (FACSO): Esto le da una mirada interdisciplinaria, donde se destaca la formación crítica, reflexiva, autónoma y valórica, en el ejercicio ético de la profesión y en coherencia con los enfoques de sustentabilidad, derechos humanos y responsabilidad social. (Facultad de Ciencias Sociales, 2015, p. 19-20) 
- Duración de la carrera: Tiene la duración de cinco años, ya que se ha elaborado un plan de estudios donde se aborde en profundidad contenidos esenciales para poder ejercer como educadora de párvulos con niños y niñas en ambos ciclos curriculares ( 0 a 3 años y 3 a 6 años); además, abordar temas como las construcciones sociales de la infancia, la inclusión y diversidad, las transiciones entre Educación Parvularia y Básica, la investigación pedagógica, gestión curriculares, diferentes disciplinas y su didáctica, entre otros. Asimismo, las estudiantes al octavo semestre obtienen el grado de Licenciatura en Educación y pueden avanzar con su formación continua. Esta decisión se avala en serias investigaciones que demuestran que existe un efecto estadísticamente significativo entre la formación superior especializada y las competencias de los/as Educadores/as de Párvulos. Entre los hallazgos se evidencia que aquellos/as que cuentan con estudios superiores de al menos 4 años de duración y especializados en primera infancia, son capaces de: (a) desplegar prácticas pedagógicas más apropiadas a las características del desarrollo de los niños y niñas a su cargo, (b) demostrar mayor sensibilidad a las necesidades de éstos, y (c) influir en la calidad del centro educativo en su conjunto. (Fukkink y Lont 2007 en Universidad de Chile, 2014, p. 20-21)

- Actividades curriculares integradas: Al finalizar cada ciclo se espera establecer hitos evaluativos (integrados entre las actividades curriculares) que den cuenta de los marcos complejos que propicia un currículo orientado al desarrollo de competencias

- Programa estructurado en ciclos: ciclo de formación Inicial; éste contempla cuatro semestres, habilita para la obtención de una certificación intermedia, si el estudiante ha alcanzado las competencias necesarias para este fin y ha completado todas las actividades curriculares requeridas., ciclo de Formación Intermedia; contempla cuatro semestres, habilita para la obtención de la Licenciatura y finalmente, el Ciclo de Formación Profesional que está conformado por dos 
semestres, comprende las actividades de titulación y conduce a la obtención del Título Profesional.

- Prácticas desde el primer semestre y en progresión: Las prácticas se constituyen la “columna vertebral” del plan de fomación, teniendo una articulación vertical y horizontal con las diferentes asignatura. Todas son obligatorias y con prerrequisitos. El ciclo inicial organiza sus actividades curriculares con una mirada de “aproximación al campo pedagógico”, a fin de sentar las bases para la formación profesional e instalar la reflexión permanente sobre su quehacer. Asimismo, el ciclo intermedio articula las actividades curriculares a partir de la reflexión sobre la “experiencia pedagógica y los saberes profesionales” con el objeto de problematizar la propia práctica y levantar saberes. Finalmente, el ciclo profesional orienta el desarrollo de actividades curriculares hacia la “docencia y la reflexión pedagógica”, propiciando que el ejercicio profesional se vincule en la generación de conocimientos a partir del propio quehacer. Cabe mencionar que existe en la propuesta formativa articulación entre práctica profesional, seminarios de investigación y talleres de práctica por cuanto, las estudiantes tendrán media jornada lectiva de Práctica Profesional (terreno) y el resto de la jornada se espera realicen los cursos anteriormente mencionados.

- Formación continua. El plan de estudio que se propone contempla articulación temprana de los ciclos formativos que constituyen el diseño curricular del Pregrado de la Universidad de Chile. De esta manera, la carrera de Pedagogía en Educación Parvularia establece cuatro años para la obtención del grado de Licenciatura en Educación y un quinto año articulable con la educación continua (posgrado) y conducente al título profesional.

\section{Énfasis Pedagógico:}

- Profesional de la educación: Con sólidas habilidades para argumentar la relevancia de la Educación Parvularia, comprendiendo y valorando este tramo 
etáreo como un período determinante para las posibilidades y el desarrollo integral de las personas. En tal sentido se le ofrecerán herramientas para que vincule la teoría y práctica, en forma permanente.

- Producción de conocimiento pedagógico: Como parte integrante de la Facultad de Ciencias sociales, la /el Educadora/or desarrollará una sólida capacidad investigativa, a partir de la observación, la indagación y la reflexión sobre la propia práctica, será capaz de generar conocimiento pertinente a su ámbito de desempeño, siendo parte esencial de los sucesos que ocurren diariamente en el espacio educativo analizando y proponiendo desde su práctica misma nuevos desafíos, posesionándose como investigadores educacionales; testigos presenciales de hechos y/o fenómenos ocurridos en el contexto educacional aportando a la generación de conocimiento pedagógico.

- Análisis Crítico: la /el Educadora/or desarrollará, problematizará el conocimiento del área educativa con miras a aportar, expresándose a través de habilidades de indagación, análisis y comunicación, promoviendo la resolución de problemas a los que se enfrenta en su práctica y rompiendo con la mirada reproduccionista, hegemónica y homogénea muchas veces desarrolladas en las prácticas pedagógicas.

- Compromiso Social: respondiendo a la misión al de la Universidad, el plan de formación intenciona un compromiso de equidad e inclusión en el quehacer de las /los futuros profesionales de la Educación Parvularia, propiciando la capacidad de reconocer, valorar y desarrollarse en diversos contextos y entregando herramientas para el empoderamiento de un rol que promueva la transformación.

- Trabajo con la comunidad: con un fuerte énfasis en el trabajo multidisciplinario, la/el profesional de la Educación Parvularia tendrá la capacidad de liderar equipos y constituir redes tanto con la familia como con la comunidad y con otras instituciones relativas a su quehacer pedagógico. 


\section{LA EDUCACION PARVULARIA EN LA UNIVERSIDAD DE CHILE: UN COMPROMISO PAÍS}

- Rango de atención y modalidades: la formación recibida le permitirá ejercer con niños/as desde el nacimiento hasta los seis años, ello en diferentes modalidades curriculares y modalidades de atención tanto convencionales como no convencionales o alternativos. 


\section{LA EDUCACION PARVULARIA EN LA UNIVERSIDAD DE CHILE: UN COMPROMISO PAÍS}

\section{AVANCES Y DESAFIOS:}

Hoy contamos con un grupo de educadoras de párvulos en formación que escogieron esta carrera en la Universidad de Chile y un cuerpo académico comprometido con la Educación Parvularia, compuesto por docentes de diferentes disciplinas, principalmente del Departamento de Educación y de la Facultad de Ciencias Sociales, además de profesores invitados de otras unidades académicas de la Universidad o de Instituciones que permiten tener una mirada diversa, profunda y crítica frente a diversos temas contingentes y relevantes de la Educación Parvularia.

Hemos constituido el Consejo Asesor de la Carrera, convocando a un conjunto de expertos en primera infancia de nuestro país de las más importantes instituciones y organizaciones que trabajan por la infancia: UNESCO, UNICEF, SENAME, Organización de Estados Iberoamericanos (OEI), Ministerio de Educación, Junta Nacional de Jardines Infantiles, Fundación Integra, Sistema Chile Crece Contigo, Organización Mundial de Educación Preescolar (OMEP), Colegio de Educadoras de Párvulos A.G., el Instituto Internacional de Educación Infantil, de la Universidad Central, y el Liceo Experimental Manuel de Salas. Dicha decisión se pensó con la convicción de la necesidad de una constante retroalimentación entre el quehacer universitario y las distintas instancias y organismos públicos y privados dedicados a la educación de la primera infancia, lo que permite recibir sugerencias y propuestas para mejorar de forma permanente la calidad del proyecto formativo y además, relacionarse permanentemente la academia con la política pública y las instituciones cuyo objetivo es la primera infancia.

Como desafíos sabemos que debemos de seguir construyendo, sistematizando y evaluando el trabajo desarrollado de manera de desarrollar los ajustes que fueran necesarios. Otro desafío como carrera y como país es ofrecer una carrera atractiva y campo laboral que atraiga a mayor número de postulantes y ente ellos esperamos a varones, los que sabemos podrían ser importantes agentes educativos, pero para ello debemos de seguir avanzando como sociedad despejando prejuicios y ofreciendo mejores condiciones laborales. 
Continuar aportando a las necesidades de nuestro país en torno a las transiciones y articulación entre niveles creemos que es un desafío y tarea pendiente por la que trabajaremos. Por ello, otro desafío es continuar con la relación permanente entre las pedagogías que se ofrecen en la Universidad de Chile, de manera que nuestros educadores que egresen permitan favorecer este nudo en el sistema, así como aportarán desde su mirada crítica y social a defender una educación más justa, más pertinente y con mejores oportunidades de aprendizaje a los niños y niñas en un ambiente de profundo respeto hacia a los niños, niñas, familias y comunidades en las que desempeñen.

\section{CITAS:}

(1) El equipo que trabajó diseñando la propuesta de carrera Pedagogía en Educación Parvularia estuvo integrado por:

- Francisca Duarte. Representante Asamblea Estudiantil Carrera de Educación Parvularia y Básica Inicial, Universidad de Chile.

- Nayira Belmar Montano. Representante Asamblea Estudiantil Carrera de Educación Parvularia y Básica Inicial, Universidad de Chile.

- Dra. Valeria Herrera Fernández, profesora en Educación Diferencial. Investigadora CIAE-FACSO, actualmente forma parte del Depto. de Educación de la Universidad de Chile.

- Dra. Daniela S. Jadue Roa. Educadora de Párvulos y Básica, CIAE, Universidad de Chile. Asesora académica de la Comisión.

- Dr. Mauricio Núñez Rojas. Académico Departamento de Estudios Pedagógicos, Facultad de Filosofía y Humanidades. Universidad de Chile.

- Mg. Marcela Pardo Quiñones. Investigadora CIAE, Universidad de Chile. Coordinadora de la Comisión.

- Mg. Angélica Riquelme Arredondo. Educadora de Párvulos. Académica del Departamento de Educación.

- Dra. Alejandra Silva Machefert, Educadora de Párvulos, Académico Departamento de Estudios Pedagógicos, Facultad de Filosofía y Humanidades. Universidad de Chile. 


\section{LA EDUCACION PARVULARIA EN LA UNIVERSIDAD DE CHILE: UN COMPROMISO PAÍS}

- Mg. María José Troncoso Álvarez. Educadora de Párvulos en ejercicio.

- Ana Moraga Tononi. Profesional de la Red para la Excelencia Docente de la Universidad de Chile, RED.

- Francisca Corvalán Profesional de la Red para la Excelencia Docente de la Universidad de Chile, RED.

- María José Opazo Pérez, Antropóloga Universidad de Chile. Profesional como secretaría Ejecutiva.

- Natalia Yáñez Ruiz. Socióloga Universidad de Chile. Profesional como secretaría Ejecutiva

- Mónica Manhey Moreno. Educadora de Párvulos, Académica Departamento de Educación, FACSO. 


\section{REFERENCIAS BIBLIOGRÁFICAS:}

Bisschop, J. (2006)

Manual para la implementación y uso de los créditos en la educación musical de nivel superior. Traducido por Álvaro Cabrera Maray

FACSO (2015) Facultad de Ciencias Sociales, Escuela de Pregrado. Científico/a Social de la Facultad de Ciencias Sociales de la Universidad de Chile. Informe II (2015) Presentación Propuesta de Sello. p. 19-20

Peralta, M.V. (2013) Desde “expósitos” a personas-sujetos de sus aprendizajes: El lento transitar de los bebés latinoamericanos al derecho de una educación oportuna y pertinente desde el nacimiento. Universidad Central. Santiago. pp. 123-124.

Serrano, Sol. (1993) Universidad y nación", Santiago de Chile, Editorial Universitaria. http://www.uchile.cl/portal/presentacion/histori a/resena-historica/4727/una-mirada-a-la-historia. Descargado el 15 abril 2015.

Universidad de Chile. (2010). Modelo educativo 2010. Santiago, Chile.

Universidad de Chile (2012. Plan de Mejoramiento Institucional. "Primera Fase de Implementación Proyecto Institucional de Educación de la Universidad de Chile: Formando Profesores para Chile”. P.12

Universidad de Chile, (2014). Formulario de Presentación de Nuevos Programas de Pregrado: Pedagogía en Educación Parvularia Facultad de Ciencias Sociales. p. 20-21)

Universidad de Chile, (2015). Modelo educativo de la Universidad de Chile. Santiago, Chile: Ediciones Universidad de Chile. p.10 